\title{
ANÁLISE E DESENVOLVIMENTO DE
}

\section{MATERIAIS CERÂMICOS:}

\section{NOVAS PERSPECTNAS}

\section{Organizadores:}

Aluska do Nascimento Simões Braga

Geysivana Késsya Garcia Carvalho Hitalo de Jesus Bezerra da Silva José Rosa de Souza Farias Valdeci Bosco dos Santos Veruska do Nascimento Simões

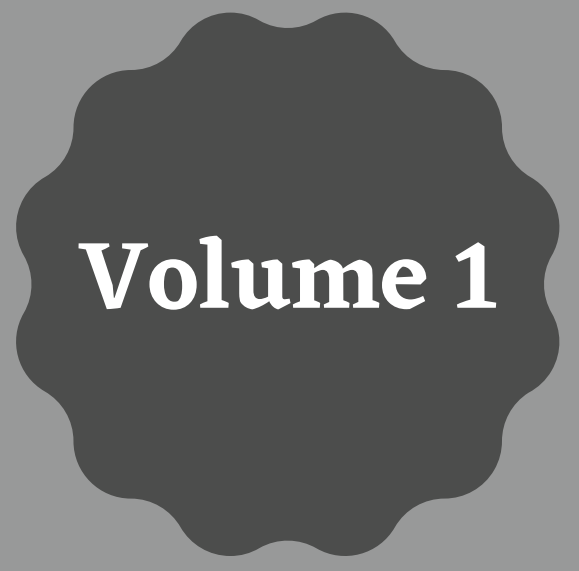
Ycaro Breno Alves de Almeida

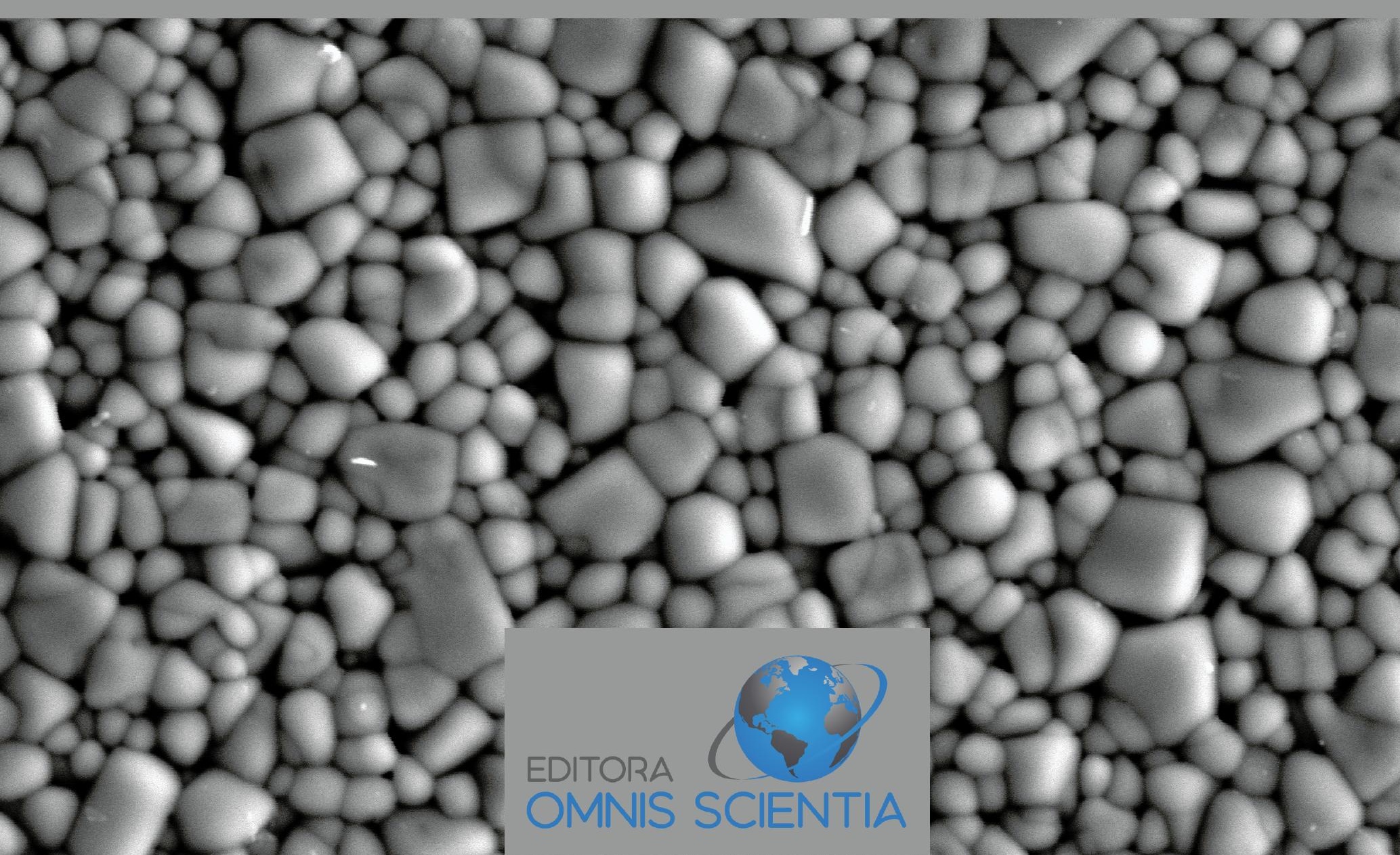




\section{ANÁLISE E DESENVOLVIMENTO DE}

\section{MATERIAIS CERÂMICOS:}

\section{NOVAS PERSPECTNAS}

\section{Organizadores:}

Aluska do Nascimento Simões Braga Geysivana Késsya Garcia Carvalho Hitalo de Jesus Bezerra da Silva José Rosa de Souza Farias Valdeci Bosco dos Santos Veruska do Nascimento Simões

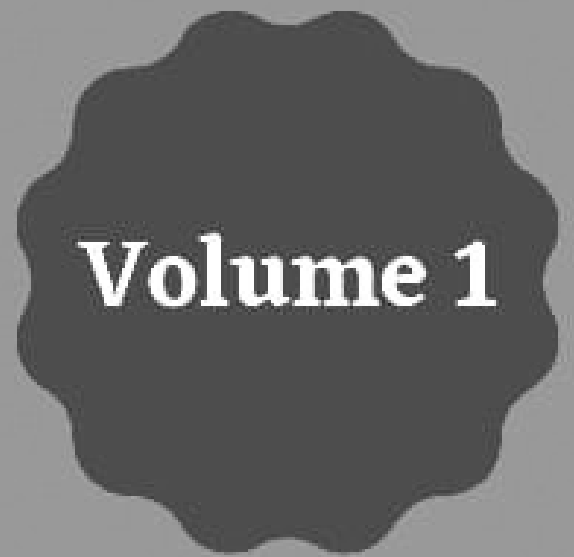
Ycaro Breno Alves de Almeida

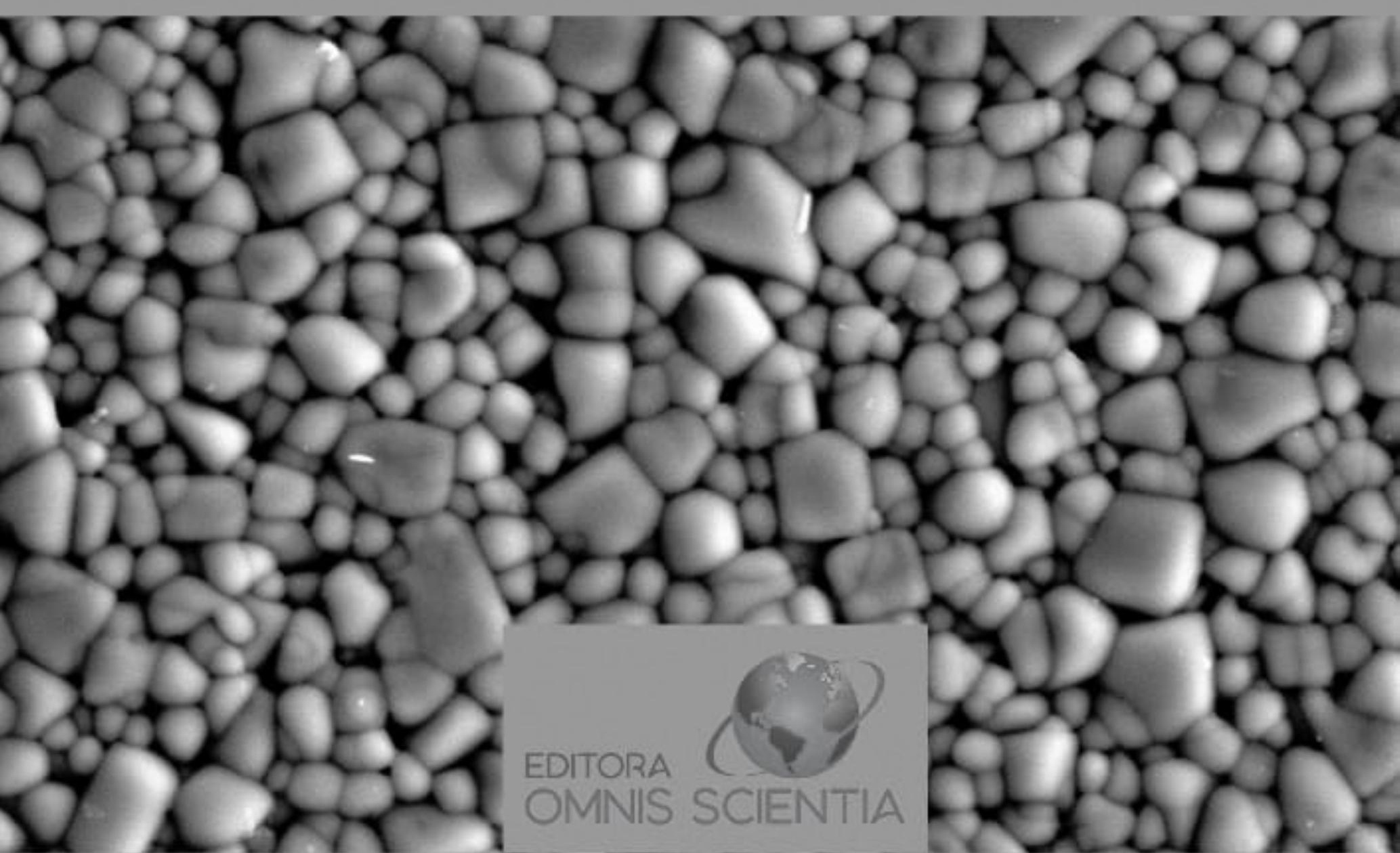


Editora Omnis Scientia

ANÁLISE E DESENVOLVIMENTO DE MATERIAIS CERÂMICOS:

NOVAS PERSPECTIVAS

Volume 1

$1^{\mathrm{a}}$ Edição

TRIUNFO - PE

2022 


\section{Editor-Chefe}

Me. Daniel Luís Viana Cruz

\section{Organizadores}

Aluska do Nascimento Simões Braga

Geysivana Késsya Garcia Carvalho

Hitalo de Jesus Bezerra da Silva

José Rosa de Souza Farias

Valdeci Bosco dos Santos

Veruska do Nascimento Simões

Ycaro Breno Alves de Almeida

\section{Conselho Editorial}

Dr. Cássio Brancaleone

Dr. Marcelo Luiz Bezerra da Silva

Dra. Pauliana Valéria Machado Galvão

Dr. Plínio Pereira Gomes Júnior

Dr. Walter Santos Evangelista Júnior

Dr. Wendel José Teles Pontes

\section{Editores de Área - Engenharias}

Dra. Elba Gomes dos Santos Leal

Dr. Mauro de Paula Moreira

\section{Assistente Editorial}

Thialla Larangeira Amorim

\section{Imagem de Capa}

Os autores

\section{Edição de Arte}

Vileide Vitória Larangeira Amorim

\section{Revisão}

Os autores

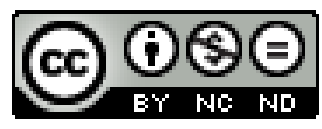

Este trabalho está licenciado com uma Licença Creative Commons - AtribuiçãoNãoComercial-SemDerivações 4.0 Internacional.

O conteúdo abordado nos artigos, seus dados em sua forma, correção e confiabilidade são de responsabilidade exclusiva dos autores. 
Dados Internacionais de Catalogação na Publicação (CIP) (eDOC BRASIL, Belo Horizonte/MG)

A532 Análise e desenvolvimento de materiais cerâmicos [livro eletrônico] : novas perspectivas / Organizadores Aluska do Nascimento Simões Braga... [et al.]. - Triunfo, PE: Omnis Scientia, 2022. 74 p. : il.

Inclui bibliografia

ISBN 978-65-88958-77-3

DOI 10.47094/978-65-88958-77-3

1. Materiais cerâmicos. 2. Cerâmicas tradicionais. 3. Cerâmicas avançadas. 4. Engenharia de materiais. I. Braga, Aluska do Nascimento Simões. II. Carvalho, Geysivana Késsya Garcia. III.Silva, Hitalo de Jesus Bezerra da. IV. Farias, José Rosa de Souza. V.Santos, Valdeci Bosco dos. VI. Simões, Veruska do Nascimento. VII. Almeida, Ycaro Breno Alves de.

CDD 620.11

Elaborado por Maurício Amormino Júnior - CRB6/2422

\author{
Editora Omnis Scientia \\ Triunfo - Pernambuco - Brasil \\ Telefone: +55 (87) 99656-3565 \\ editoraomnisscientia.com.br \\ contato@editoraomnisscientia.com.br
}




\section{PREFÁCIO}

Materiais cerâmicos são uma classe de materiais que abrange uma grande variedade de substâncias naturais e sintéticas, tais como tijolos, revestimentos, pedras, concreto, abrasivos, vidrados para porcelana, isolantes, dielétricos, materiais magnéticos e não-metálicos, refratários para altas temperaturas e muitos outros. Popularmente, o termo cerâmica é utilizado para designar objetos de arte feitos a partir de massas plásticas cerâmicas. Usualmente, os materiais cerâmicos podem ser descritos como compostos inorgânicos formados por elementos metálicos e não metálicos. Visando melhor compreensão dos materiais cerâmicos, costuma-se dividi-los em dois grupos básicos: as cerâmicas tradicionais e as cerâmicas avançadas.

As cerâmicas tradicionais compreendem todos os materiais cerâmicos fabricados a partir de matérias-primas que são encontrados na natureza, como argilas, cerâmicas vermelhas, cerâmicas brancas, entre outros. Os produtos das cerâmicas tradicionais incluem: telhas, tijolos, cimento, revestimentos cerâmicos (pisos e azulejos), louças de mesa, vidros em geral, materiais refratários e isoladores elétricos.

As cerâmicas avançadas, também conhecidas como cerâmicas de alta tecnologia, diferem das tradicionais em virtude do controle mais rígido dos materiais empregados e nas etapas de processamento, o que influi nas propriedades e na microestrutura do material. São compostas por materiais sintéticos de alto grau de pureza, tais como o óxido de alumínio (A12O3), carboneto de silício ( $\mathrm{SiC}$ ), nitreto de silício ( $\mathrm{Si3N} 4)$ e óxido de zircônio ( $\mathrm{ZrO} 2)$, entre outros, preparados por métodos mais sofisticados, envolvendo processos controlados, bem como melhor entendimento a nível microscópico. Os produtos das cerâmicas avançadas incluem: cerâmicas eletrônicas (isolantes e semicondutores), cerâmicas magnéticas (ferritas), cerâmicas ópticas (fibras ópticas, materiais para laser), elementos combustíveis (pastilhas de UO2), etc.

Neste sentido, este livro aborda diferentes pesquisas relacionados aos materiais cerâmicos, apresentando de maneira clara e objetiva resultados de estudos originais, bem como dados e informações pertinentes por meio de revisões bibliográficas acerca dessa área da ciência e engenharia de materiais. 


\section{SUMÁRIO}

CAPÍTULO 1

.10

INFLUÊNCIA DA RAZÃo ÁCIDO CÍTRICO/CÁTIONS METÁLICOS NA SÍNTESE DE MULITA PELO MÉTODO PECHINI

José Rosa de Souza Farias

Slanna Larissa Olimpio Costa

Ycaro Breno Alves de Almeida

Veruska do Nascimento Simões

Geysivana Késsya Garcia Carvalho

Hitalo de Jesus Bezerra da Silva

Érica Karine Alves de Lima

Valdeci Bosco dos Santos

Aluska do Nascimento Simões Braga

DOI: 10.47094/978-65-88958-77-3/10-17

CAPÍTULO 2

EFEITO DO TETRAHIDROFURANO COMO SOLVENTE NA SÍNTESE DE MULITA PELO MÉTODO PECHINI

José Rosa de Souza Farias

Slanna Larissa Olimpio Costa

Ycaro Breno Alves de Almeida

Veruska do Nascimento Simões

Geysivana Késsya Garcia Carvalho

Hitalo de Jesus Bezerra da Silva

Wollia Nayane Araújo Vitoriano

Valdeci Bosco dos Santos

Aluska do Nascimento Simões Braga

DOI: 10.47094/978-65-88958-77-3/18-25 
CAPÍTULO 3

INFLUÊNCIA DE DIFERENTES ÁLCOOIS COMO SOLVENTES NASIINTESE DE MULITA

PELO MÉTODO SOL-GEL

José Rosa de Souza Farias

Slanna Larissa Olimpio Costa

Adryann Millos Santos De Freitas

Ycaro Breno Alves de Almeida

Veruska do Nascimento Simões

Geysivana Késsya Garcia Carvalho

Hitalo de Jesus Bezerra da Silva

Valdeci Bosco dos Santos

Aluska do Nascimento Simões Braga

DOI: 10.47094/978-65-88958-77-3/26-33

CAPÍTULO 4.

AVALIAÇÃO DA CITOTOXICIDADE DE CERÂMICAS BIFÁSICAS DE HAp/ß̧-TCP

Ycaro Breno Alves de Almeida

Slanna Larissa Olimpio Costa

José Rosa de Souza Farias

Diogenes de Moura Junior

Victoria Regia Alves Sales

Ketelly Estefane da Silva Alves

Genivaldo Melo da Rocha

Veruska do Nascimento Simões

Geysivana Késsya Garcia Carvalho

Hitalo de Jesus Bezerra da Silva

Valdeci Bosco dos Santos

Aluska do Nascimento Simões Braga

DOI: 10.47094/978-65-88958-77-3/34-43 
CAPÍTULO 5

SÍNTESE DE HIDROXIAPATITA VIA ROTA PECHINI: UMA BREVE REVISÃO

Ycaro Breno Alves de Almeida

Slanna Larissa Olimpio Costa

José Rosa de Souza Farias

Diogenes de Moura Junior

Victoria Regia Alves Sales

Ketelly Estefane da Silva Alves

Genivaldo Melo da Rocha

Veruska do Nascimento Simões

Geysivana Késsya Garcia Carvalho

Hitalo de Jesus Bezerra da Silva

Valdeci Bosco dos Santos

Aluska do Nascimento Simões Braga

DOI: 10.47094/978-65-88958-77-3/44-59

CAPÍTULO 6 .60

NANOFIBRAS DE MANGANITA DE LANTÂNIO DOPADAS COM ESTRÔNCIO: UMA ANÁLISE BIBLIOMÉTRICA

Érica Karine Alves de Lima

Wollia Nayane Araújo Vitoriano

Hitalo de Jesus Bezerra da Silva

Maria Elayne Rodrigues Alves

Humberto Denys de Almeida Silva

Fernanda da Luz Barbosa

José Rosa de Souza Farias

Ycaro Breno Alves de Almeida

Veruska do Nascimento Simões

Geysivana Késsya Garcia Carvalho

Aluska do Nascimento Simões Braga

Valdeci Bosco dos Santos

DOI: 10.47094/978-65-88958-77-3/60-71 
NANOFIBRAS DE MANGANITA DE LANTÂNIO DOPADAS COM ESTRÔNCIO: UMA ANÁLISE BIBLIOMÉTRICA

Érica Karine Alves de Lima';

Universidade Federal do Piauí (UFPI), Teresina, Piauí.

http://lattes.cnpq.br/8186490901865658

Wollia Nayane Araújo Vitoriano²;

Universidade Federal do Piauí (UFPI), Teresina, Piauí.

http://lattes.cnpq.br/3479562872784671

Hitalo de Jesus Bezerra da Silva ${ }^{3}$;

Universidade Federal de Pernambuco (UFPE), Recife, Pernambuco.

http://lattes.cnpq.br/4510351692039237

Maria Elayne Rodrigues Alves ${ }^{4}$;

Universidade Federal do Piauí (UFPI), Teresina, Piauí.

http://lattes.cnpq.br/6730439235097176

Humberto Denys de Almeida Silva ${ }^{5}$;

Universidade Federal do Piauí (UFPI), Teresina, Piauí.

http://lattes.cnpq.br/0272180920811513

Fernanda da Luz Barbosa ${ }^{6}$;

Instituto Federal de Educação, Ciência e Tecnologia do Piauí (IFPI), Teresina, Piauí.

http://lattes.cnpq.br/3563671971194880

José Rosa de Souza Farias';

Universidade Federal do Piauí (UFPI), Teresina, Piauí.

http://lattes.cnpq.br/9404467330103347

Ycaro Breno Alves de Almeida ${ }^{8}$;

Universidade Federal do Piauí (UFPI), Teresina, Piauí

http://lattes.cnpq.br/4070307554924095

Veruska do Nascimento Simões';

Universidade Federal do Rio Grande do Norte (UFRN), Natal, Rio Grande do Norte

http://lattes.cnpq.br/1149592827133122 


\section{Geysivana Késsya Garcia Carvalho ${ }^{10}$;}

Universidade Federal do Piauí (UFPI), Teresina, Piauí

http://lattes.cnpq.br/0242153749540840

\section{Aluska do Nascimento Simões Braga ${ }^{11}$;}

Universidade Federal do Piauí (UFPI), Teresina, Piauí.

Instituto Federal de Educação, Ciência e Tecnologia do Piauí (IFPI), Teresina, Piauí. http://lattes. cnpq.br/2429557575387821

Valdeci Bosco dos Santos ${ }^{12}$.

Universidade Federal do Piauí (UFPI), Teresina, Piauí.

Instituto Federal de Educação, Ciência e Tecnologia do Piauí (IFPI), Teresina, Piauí.

http://lattes.cnpq.br/0011700686113389

RESUMO: Cerâmicas perovskitas compostas por manganita de lantânio dopada com estrôncio (LSM) têm atraído muita atenção devido às suas excelentes características elétricas, magnéticas e estruturais. Recentemente tem se destacado como candidatas potenciais a área biomédica (hipertermia magnética, ressonância magnética e liberador de fármacos). Nanofibras apresentam diâmetro pequeno e alta área superficial. Isto as tornam muito interessantes para aplicações da nanotecnologia em vários campos da ciência/indústria em comparação aos pós nano/micrométricos e monólitos. O objetivo desse trabalho foi investigar o qualitativo de publicações sobre as nanofibras de LSM. As bases de artigos científicos pesquisadas foram: Scopus, Web of Science e Scielo. Foram utilizadas as seguintes palavras-chave: lanthanum and manganite, lanthanum and manganite and nanofiber, lanthanum and manganite and nanofiber and strontium. Por meio desta metodologia de busca foram obtidos somente 8 artigos científicos. Nenhum artigo pertinente ao tema proposto foi encontrado na base Scielo. Nessas referências, por exemplo, nanofibras de LSM demonstraram maior sensibilidade e resposta mais rápida ao monóxido de carbono, quando aplicadas como sensores de gases. Já em comparação a monólitos de LSM, as nanofibras contribuíram para um aumento em torno de $70 \%$ do efeito de magnetorresistência. Constatou-se que o tema é ainda incipiente e pouco explorado. As excelentes propriedades funcionais das LSM em combinação com notória eficiência das nanofibras possuem um excelente potencial prospectivo para as diversas áreas de aplicação científicas e tecnológicas.

PALAVRAS-CHAVE: Manganita de lantânio. Estrôncio. Nanofibras. 


\begin{abstract}
Perovskite ceramics composed of strontium-doped lanthanum manganite (LSM) have attracted much attention due to their excellent electrical, magnetic and structural characteristics. Recently, it has stood out as potential candidates in the biomedical area (magnetic hyperthermia, magnetic resonance and drug release). Nanofibers have a small diameter and high surface area. This makes them very interesting for nanotechnology applications in various fields of science/industry compared to nano/micrometric powders and monoliths. The aim of this work was to investigate the qualitative of publications on LSM nanofibers. The databases of scientific articles researched were: Scopus, Web of Science and Scielo. The following keywords were used: lanthanum and manganite, lanthanum and manganite and nanofiber, lanthanum and manganite and nanofiber and strontium. Through this search methodology, 8 scientific articles were obtained. No article relevant to the proposed theme was selected in the Scielo database. In these references, for example, LSM nanofibers demonstrated greater sensitivity and faster response to carbon monoxide, when applied as gas sensors. Compared to LSM monoliths, nanofibers contributed to an increase of around $70 \%$ in the magnetoresistance effect. It was found that the theme is still incipient and little explored. The excellent functional properties of LSM in combination with the remarkable efficiency of nanofibers have an excellent prospective potential for different scientific and technological application areas.
\end{abstract}

KEY-WORDS: Lanthanum manganite. Strontium. Nanofibers.

\title{
INTRODUÇÃO
}

Nas últimas décadas, cerâmicas perovskitas da classe de óxidos mistos com configuração estrutural $\mathrm{ABO}_{3}$ (sítio A - elemento de terra rara; sítio $\mathrm{B}$ - elemento de metal de transição), tem despertado grande interesse científico e tecnológico por estarem dentre os materiais mais populares aplicados à diversas áreas industrias. Apresentam propriedades únicas, tais como alta estabilidade, excelente atividade de oxidação, propriedades adsortivas, catalíticas, ópticas, magnéticas, eletrônicas e ferroelétricas (Paulo e Hernández, 2018; Garba et al., 2019).

A manganita de lantânio com substituição parcial no sítio A por estrôncio ( $\mathrm{LSM}$ - $\mathrm{La}_{1-\mathrm{x}} \mathrm{Sr}_{\mathrm{x}} \mathrm{MnO}_{3}$ ) é um material perovskita de estrutura cristalina ortorrômbica, e conhecida por suas propriedades (elétricas, magnéticas e estruturais) interessantes. Por exemplo, é um material cátodo adequado para células combustível do tipo óxido sólido, visto sua alta condutividade elétrica e boa compatibilidade química com o eletrólito sólido de zircônia estabilizada com ítria (Tran et al., 2019; Lang et al., 2019). O dopante $\mathrm{Sr}$ na estrutura cristalina da $\mathrm{LaMnO}_{3}$ aumenta a condutividade elétrica, devido a uma mudança na razão $\mathrm{Mn}^{3+} / \mathrm{Mn}^{4+}$ (Cao et al., 2015; Xu et al., 2015). A condutividade mista das composições LSM é fortemente dependente da ligação entre íons $\mathrm{Mn}^{3+}$ e $\mathrm{Mn}^{4+}$, mas também das distâncias de $\mathrm{Mn}$ - $\mathrm{O}$ e do ângulo de ligação $\mathrm{Mn}-\mathrm{O}$ - Mn. Além disso, a interação da dupla troca e o efeito de Jahn-Teller explica o ordenamento ferromagnético que ocorre quando os elétrons são 
transportados de íons $\mathrm{Mn}^{3+}$ para íons $\mathrm{Mn}^{4+}$, através de átomos de oxigênio, que as torna um material com condutibilidade elétrica especial (Cao et al., 2015; Burrola-Gándara et al., 2019). LSM também exibe o efeito magnetorresistência colossal (Dwivedi et al., 2015; Karwoth et al., 2019), o que propicia um dispositivo com caráter magnético soft (Karwoth et al, 2019).

Recentemente, outras novas aplicações têm se revelado. $\mathrm{La}_{1-\mathrm{x}} \mathrm{Sr}_{\mathrm{x}} \mathrm{MnO}_{3}$ tem atraído atenção como um fotocatalítico potencial ao meio ambiente, de baixo custo e com atividade em luz visível (Afje e Ehsani, 2018; Turky et al., 2017). Estudos preliminares sobre as nanopartículas magnéticas LSM, tem demonstrado que estas são candidatas promissoras no campo biomédico (hipertermia magnética, agente de contraste de ressonância magnética e liberador de fármacos acionada por ímã). Apresentam uma alta magnetização para tais aplicações e sua temperatura da Curie varia entre 6,85 e $86,85^{\circ} \mathrm{C}$, a depender da quantidade de $\mathrm{Sr}$ (Kumar et al., 2019; Chiabrera et al., 2017).

Existem diferentes métodos de preparação de nanomateriais, os quais utilizam diferentes precursores, e consequentemente resultam em distintos tamanhos e morfologias de nanopartículas, bem como em diferentes propriedades funcionais. Desta forma, as nanopartículas de materiais perovskitas à base $\mathrm{LaMnO}_{3}$ têm sido frequentemente sintetizadas por métodos tais como: sol gel (Wu et al., 2005), método de citrato (Choi et al., 2018), combustão (Turky et al., 2017), co precipitação (Turky et al., 2018) e síntese hidrotermal (Hehab et al., 2019)

É bem conhecido que o controle do tamanho, forma e estrutura dos materiais nanométricos é de fundamental importância para suas aplicações da nanotecnologia. Recentemente, a produção de nanomateriais cerâmicos unidimensionais (nanofibras) tem atraído atenção por diversos pesquisadores, não somente pelos pequenos diâmetros (10-100 nm) das fibras, mas também por sua área superficial elevada, baixa densidade e estrutura porosa (Panda e Sahoo, 2013; Zhi et al., 2012). São algumas das características muito interessantes que tornam as nanofibras com maior potencial para aplicações funcionais em vários campos da ciência/indústria em comparação aos pós em escala nanométrica (ou micrométrica). Algumas nanofibras cerâmicas (perovskitas ou não) que têm sido sintetizadas, incluem $\mathrm{BaTiO}_{3}$ (Zhuang et al, 2018), $\mathrm{LaFeO}_{3}$ (Mun et al, 2020), $\mathrm{Al}_{2} \mathrm{O}_{3}$ (Muzenski et al, 2020) dentre outras.

As publicações científicas são instrumentos capazes de destacar o atual desenvolvimento científico e tecnológico, e reconhecer os potenciais de produtos para diversas aplicações. Diante do exposto, a presente pesquisa teve por objetivo investigar o qualitativo de publicações disponíveis nas bases de documentos científicos (Scopus, Web of Science e Scielo), considerando os estudos sobre nanofibras de manganita de lantânio $\left(\mathrm{LaMnO}_{3}\right)$ dopada com estrôncio.

\section{METODOLOGIA}

O presente estudo foi realizado através de uma prospecção científica, tendo como base o levantamento do número de artigos científicos publicados sobre a temática "nanofibras de manganita de lantânio dopadas com estrôncio", através de mecanismos de busca em bases especializadas. Para tanto, utilizou-se as bases de periódicos Scopus, Web of Science e Scielo. As combinações de 
palavras empregadas foram, em inglês, "lanthanum and manganite", "lanthanum and manganite and nanofiber", "lanthanum and manganite and nanofiber and strontium", nos campos de título e/ou resumo. Não foram utilizados mecanismos de exclusão para a busca, de modo que todos os resultados reportados pelas bases até março de 2021, momento da pesquisa, foram considerados neste estudo.

\section{RESULTADOS E DISCUSSÕES}

A Tabela 1 apresenta a relação e os resultados reportados pelas bases de artigos. Verificase, inicialmente, elevados valores em relação ao número de resultados reportados, especificamente para as bases Scopus e Web of Science, e quando relacionadas às palavras-chave "lanthanum" e "manganite". Após refinamento, nota-se que o número de resultados decai, pois os termos da busca saem de uma ótica mais abrangente, para outra mais específica. Desta forma, a terceira combinação de palavras "lanthanum", "manganite", "nanofiber" e "strontium", foi considerada por melhor retratar o escopo da presente pesquisa.

Tabela 1. Relação entre palavras-chave e resultados reportados pelas bases de documentos científicos.

\begin{tabular}{|c|c|c|c|}
\hline Palavras-Chave & Scopus & Wef of Science & Scielo \\
\hline lanthanum and manganite & 4309 & 2018 & 12 \\
\hline lanthanum and manganite and nanofiber & 12 & 3 & 0 \\
\hline $\begin{array}{l}\text { lanthanum and manganite and nanofiber } \\
\text { and strontium }\end{array}$ & 6 & 2 & 0 \\
\hline
\end{tabular}

Fonte: Autoria própria, 2021.

A Figura 1 ressalta o quantitativo de artigos distribuídos entre as três bases consultadas, considerando-se a terceira combinação de palavras-chave. Assim sendo, observa-se que a base Scopus reportou o total de 6 documentos, enquanto que para a Web of Science, foram reportados apenas 2 artigos. Não foi encontrado nenhum resultado para a busca na base Scielo. 
Figura 1. Distribuição de artigos depositados por base de periódicos.

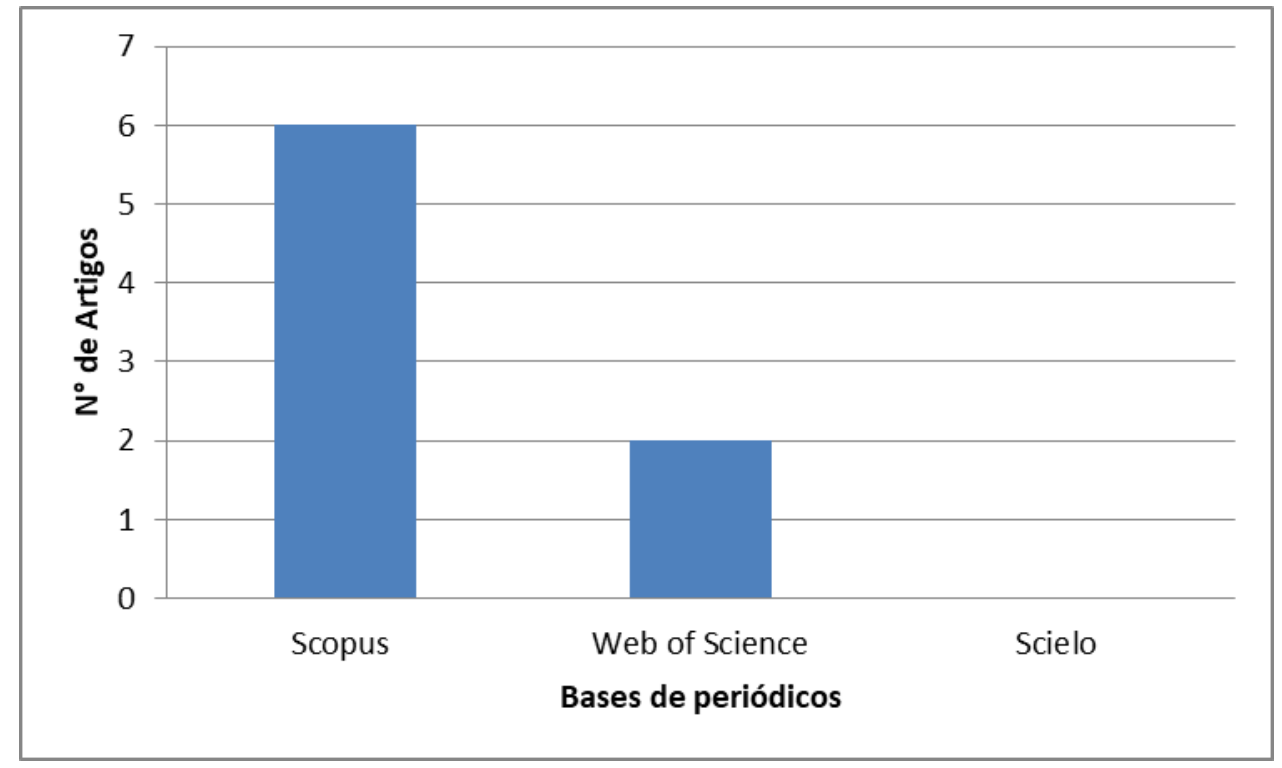

Fonte: Autoria própria, 2021.

A distribuição de artigos publicados ao longo dos anos (2012 a 2019) está apresentada na Figura 2. É notável a baixa produção científica sobre a temática no período. Observa-se que o primeiro artigo reportado foi publicado em 2012, de autoria de Zhi et al. (2012), o qual apresenta a fabricação de eletrodos de detecção de um sensor eletroquímico para detecção de monóxido de carbono $(\mathrm{CO})$ em altas temperaturas. Para este sensor, foi utilizado as nanofibras de $\mathrm{LaMnO}_{3}$ dopadas com estrôncio, demonstrando possuir maior sensibilidade e resposta mais rápida ao $\mathrm{CO}$ em relação aos demais sensores. Nos anos seguintes, não se observou grandes mudanças, visto que em 2013 não houve publicações, e entre 2014 e 2015 apenas 2 registros para cada ano. Entre 2016 e 2019, nota-se a ocorrência de pelo menos uma publicação por ano, com exceção a 2017, quando não houve publicações. 
Figura 2. Distribuição de artigos por ano de publicação.

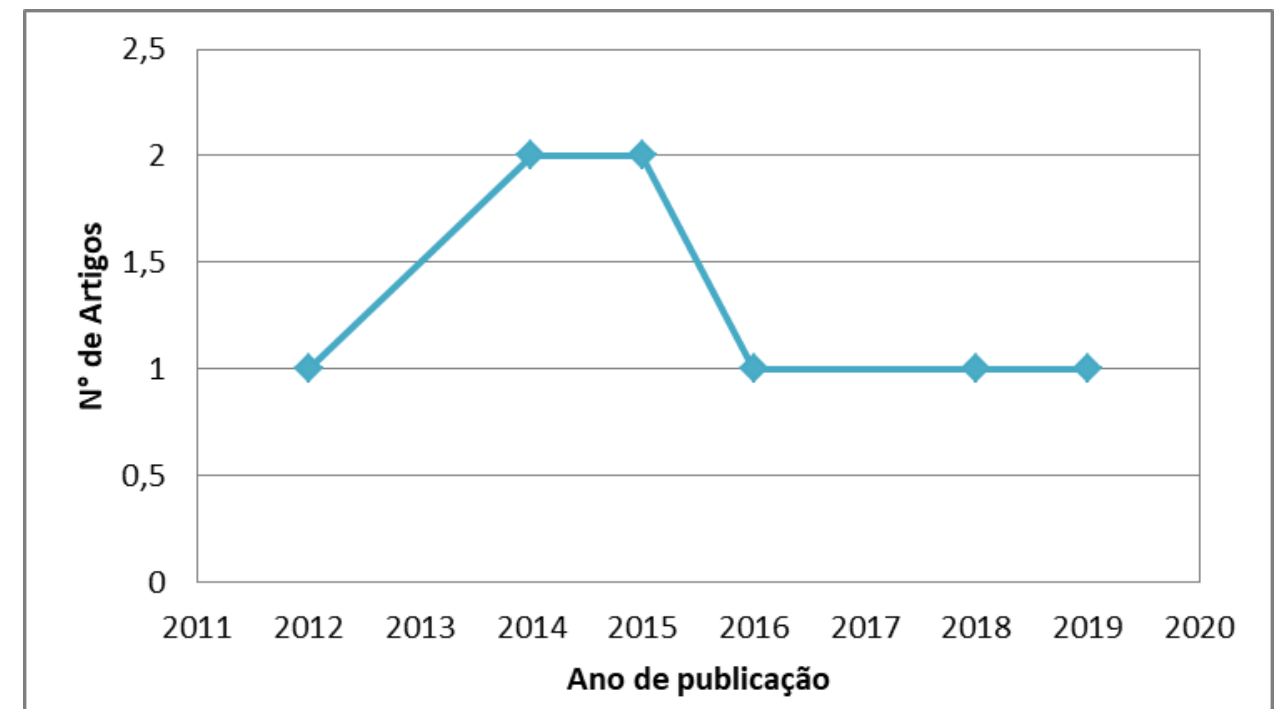

Fonte: Autoria própria, 2021.

O último artigo reportado foi publicado em 2019, de autoria de Burrola-Gándara et al. (2019), o qual faz um estudo sobre o comportamento do Spin Glass em nanofibras de $\mathrm{La}_{0.7} \mathrm{Ca}_{0.23} \mathrm{Sr}_{0 \cdot 07} \mathrm{MnO}_{3}$ obtidas por eletrofiação. Os resultados revelaram nanofibras em estado aglomerado, mostrando diâmetros médios de fibra de 75, 94 e $97 \mathrm{~nm}$ para as amostras tratadas termicamente a 699,85, 799,85 e $899,85^{\circ} \mathrm{C}$, respectivamente. Após medidas magnéticas, as amostras apresentaram valores de temperaturas de Curie iguais a $23,85,31,85$ e $40,85^{\circ} \mathrm{C}$, de acordo com os tratamentos térmicos aplicados. Além disso, um estado vítreo foi induzido pela aglomeração das nanofibras. A rotação do vidro e as temperaturas irreversíveis diminuíram com o aumento do campo magnético, e um estado altamente anisotrópico foi evidenciado para todas as amostras. Os autores concluem que o comportamento termomagnético nestas amostras de manganitas mostrou-se significativamente influenciada pela estrutura unidimensional, bem como pela variação de tamanho das nanofibras promovida pelo método de preparação, pode atuar para ajustar as propriedades magnéticas. Nesse sentido, percebe se que a produção de artigos publicados ao longo dos anos é incipiente, sem crescimento expressivo desde a primeira publicação, somando apenas poucos estudos.

Analisou-se ainda a distribuição de publicações por país de origem (Figura 3), na qual destacase a China, com 2 artigos publicados. O primeiro trata da morfologia e propriedades eletroquímicas de nanofibras obtidas com manganita e estrôncio (Cao et al., 2015), e o outro se refere à produção de um biossensor para a detecção de glicose (Xu et al., 2015). Cada um dos demais países publicou apenas um único artigo. 
Figura 3. Distribuição de artigos publicados por países.

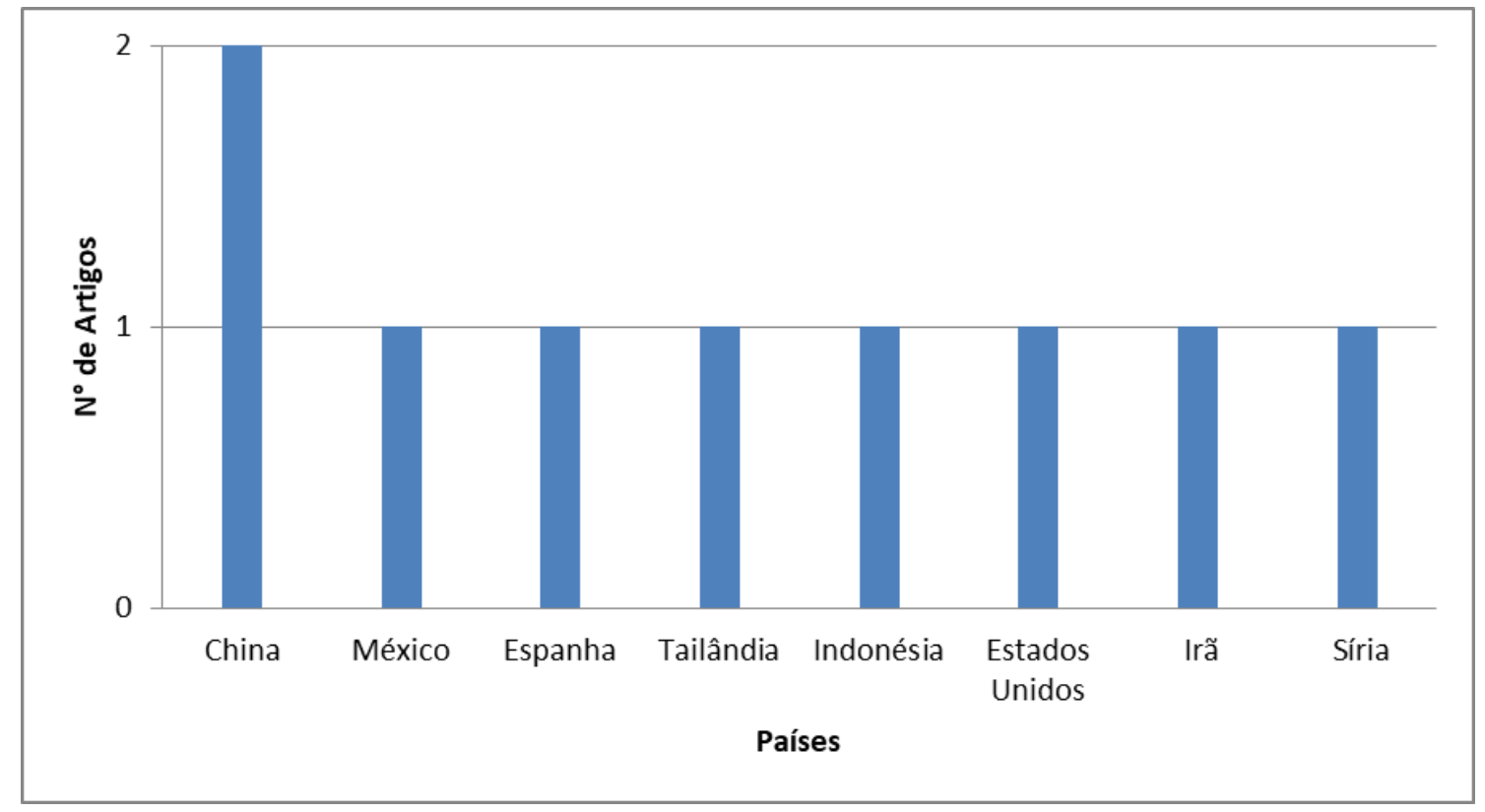

Fonte: Autoria própria, 2021.

Percebe-se que apesar das potenciais propriedades da $\mathrm{LaMnO}_{3}$ dopada com estrôncio anteriormente descritas, o tema ainda é pouco explorado pelos pesquisadores ao redor do mundo. É importante ressaltar que os cerâmicos $1 \mathrm{D}$, de fato, tendem a aumentar as propriedades das diversas aplicações. Por exemplo, nanofibras de LSMO $\left(\mathrm{La}_{0,8} \mathrm{Sr}_{0,2} \mathrm{MnO}_{3}\right)$, promoveram um aumento de aproximadamente $70 \%$ do efeito de magnetorresistência (em baixas temperaturas e campo magnético de 10 T) em comparação a monólitos de mesma composição (LSMO) (Karwoth et al, 2019).

O levantamento bibliométrico também apontou as vertentes do conhecimento que se relacionam com a temática proposta e sua interdisciplinaridade. Logo, a Figura 4 apresenta as principais áreas nas quais estão concentrados os artigos publicados sobre $\mathrm{LaMnO}_{3}$ dopada com estrôncio. 
Figura 4. Distribuição de artigos por área de publicação.

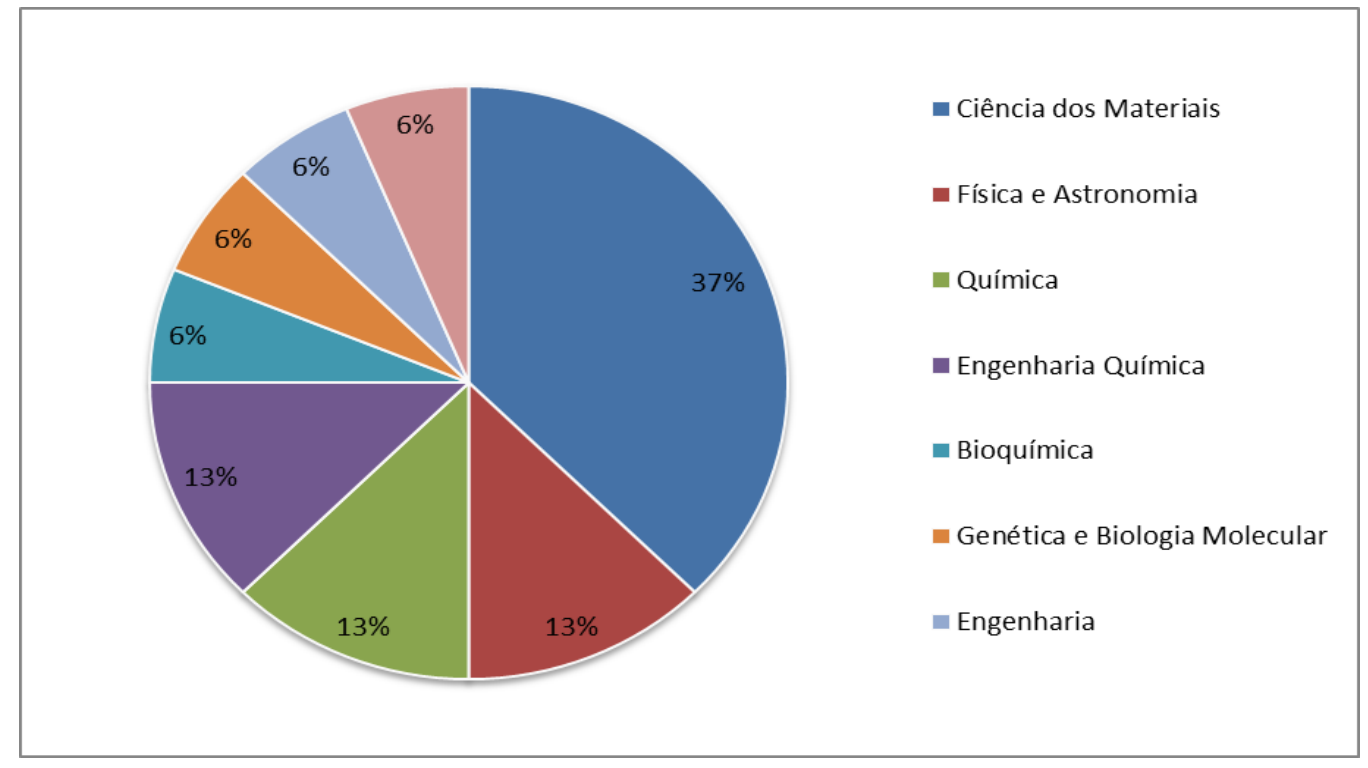

Fonte: Autoria própria, 2021.

Assim sendo, observa-se que a principal área de publicação é de Ciência de Materiais, a qual engloba 37\% dos artigos encontrados. O destaque para a concentração de publicações nesta área é em decorrência da sua multidisciplinariedade, a qual está presente em vários campos de tecnologias. Logo é possível relacionar a estrutura/nanoestrutura das fibras à base de manganitas com suas propriedades, por exemplo. Desta forma, pode-se propor o aperfeiçoamento das propriedades já bem estabelecidas ou encontrar novas propriedades/aplicações. Outras três áreas destacadas como Física e Astronomia, Química, e Engenharia Química, representam, cada uma, 13\% de artigos publicados. As demais áreas (Bioquímica, Genética e Biologia Molecular, Engenharias, Metalurgia e Engenharia Metalúrgica) representam apenas $6 \%$ dos artigos publicados, cada uma.

\section{CONCLUSÃO}

Os resultados obtidos através das bases de periódicos utilizadas revelaram que a produção científica sobre a temática é recente e contabiliza pouquíssimos artigos publicados, de modo que não foi observado crescimento significativo de publicações ao longo dos anos, desde a primeira publicação, em 2012. Semelhantemente, é evidente a inexpressividade dessa produção científica quando analisada em relação aos países de origem. Com exceção da China, que possui dois artigos publicados, observa-se apenas um artigo publicado por cada um dos 8 países reportados pela busca. Isto comprova que a temática proposta é incipiente e ainda pouco estudada, apesar de seu evidente potencial tecnológico.

Ressalta-se que as características eletromagnéticas, químicas e morfológicas, destacadas pela literatura, podem ser exploradas em diversas aplicações, sendo capazes de englobar diversas áreas de pesquisa por sua multidisciplinaridade, com destaque para a área de Ciência dos Materiais. 
Portanto, acredita-se que o interesse sobre o desenvolvimento de nanofibras cerâmicas de $\mathrm{LaMnO}_{3}$ dopada com estrôncio pode trazer grandes avanços em pesquisa, com potencial para o aperfeiçoamento das aplicações tecnológicas já existentes e/ou desenvolvimento de novas tecnologias.

\section{AGRADECIMENTOS}

Ao Programa de Iniciação Científica Voluntária da UFPI.

\section{DECLARAÇÃO DE INTERESSES}

Nós, autores deste artigo, declaramos que não possuímos conflitos de interesses de ordem financeira, comercial, político, acadêmico e pessoal.

\section{REFERÊNCIAS}

AFGHAHI, S. S. S. et al. A new multicomponent material based on carbonyl iron/carbon nanofiber/ lanthanum-strontium-manganite as microwave absorbers in the range of 8-12 GHz. Ceramics International, v. 42, n. 8, p. 9697-9702, 2016.

AFJE, F. R; EHSANI M. H. Size-dependent photocatalytic activity of $\mathrm{La}_{0.8} \mathrm{Sr}_{0.2} \mathrm{MnO}_{3}$ nanoparticles prepared by hydrothermal synthesis. Mater. Res. Express., v. 5, 2018.

BURROLA-GÁNDARA, L. A. et al. Spin Glass Behavior in $\mathrm{La}_{0.7} \mathrm{Ca}_{0.23} \mathrm{Sr}_{0.07} \mathrm{MnO}_{3}$ Nanofibers Obtained by Electrospinning. Journal of Superconductivity and Novel Magnetism, v. 32, p. 2501$2508,2019$.

$\mathrm{CAO}, \mathrm{Y}$. et al. Structure, morphology and electrochemical properties of $\mathrm{La}_{\mathrm{x}} \mathrm{Sr}_{1-\mathrm{x}} \mathrm{Co}_{0.1} \mathrm{Mn}_{0.9} \mathrm{O}_{3-\delta}$ perovskite nanofibers prepared by electrospinning method. Journal of Alloys and Compounds, v. 624, p. 31-39, 2015.

CASADO, E. et al. A. Synthesis and characterization of $\mathrm{Sr}_{1-\mathrm{x}} \mathrm{La}_{\mathrm{x}} \mathrm{MnO}_{3} / \mathrm{SiOC}$ nanocomposites decorated with 1D nanostructures for high temperature $\mathrm{CO}_{2}$ splitting. Ceramics International, v. 44, n. 15 , p. $18585-18594,2018$.

CHIABRERA, F. et al. Insights into the enhancement of oxygen mass transport properties of strontium-doped lanthanum manganite interface-dominated thin films. Solid State Ionics, v. 299, p. 70-77, 2017.

CHOI, J. G. et al. Improved high-performance $\mathrm{La}_{0.7} \mathrm{Sr}_{0.3} \mathrm{M}_{\mathrm{x}} \mathrm{Fe}_{1-\mathrm{x}} \mathrm{O}_{3}(\mathrm{M}=\mathrm{Cu}, \mathrm{Cr}, \mathrm{Ni})$ perovskite catalysts for ortho-para. Joumal of the Korean Crystal Growth and Crystal Technology, v. 28, n. 1, p. 44-50, 2018).

DWIVEDI, G. D. et al. Low temperature magnetic and transport properties of LSMO-PZT nanocomposites. Royal Society of chemistry, v. 5, p. 30748-30757, 2015. 
GARBA, Z. N. et al. review on the preparation, characterization and potential application of perovskites as adsorbents for wastewater treatment. Chemosphere, 2019.

GRUNDY et al. Assessment of the La-Sr-Mn-O system. Computer Coupling of Phase Diagrams and Thermochemistry, v. 28, p. 191-201, 2004.

HEHAB, E. A. et al. Hydrothermal synthesis, sintering and characterization of nano La-manganite perovskite doped with Ca or Sr. Ceramics International, v. 45, n. 4, p. 4894-4901, 2019.

KARWOTH, T. et al. Magnetoresistance and structural characterization of electrospun $\mathrm{La}_{1-}$ ${ }_{x} \mathrm{Sr}_{x} \mathrm{MnO}_{3}$ nanowire network fabrics with $x=0.2$. Solid State Communications, v. 290, p. 37-41, 2019.

KUMAR, R., CHAUHAN, A., JHA, S. K., KUANR, B. K. Encapsulated lanthanum strontium manganese oxide in mesoporous silica shell: Potential for cancer treatment by hyperthermia therapy. Journal of Alloys and Compounds,v.790, p. 433-446, 2019.

LANG, X. et al. Ag nanoparticles decorated perovskite $\mathrm{La}_{0.85} \mathrm{Sr}_{0.15} \mathrm{MnO}_{3}$ as electrode materials for supercapacitors. Materials Letters, v. 243, p. 34-37, 2019.

MUN, T. et al. Resistive-type lanthanum ferrite oxygen sensor based on nanoparticle-assimilated nanofiber architecture. Sensors And Actuators B: Chemical, v. 324, p. 128-712, 2020.

MUZENSKI, S. et al. Towards Ultrahigh Performance Concrete Produced with Aluminum Oxide Nanofibers and Reduced Quantities of Silica Fume. Nanomaterials, v. 10, n. 11, p. 2291, nov. 2020.

NASCIMENTO, A. C., MOHALLEM, N. D. S. Materiais usados na constituição dos principais componentes de células a combustível de óxido sólido. Cerâmica, v. 55, p. 46-52, 2009.

PANDA, PK., SAHOO, B. Synthesis and applications of electrospun nanofibers - a review. Nanotechnology, Fundam Appl, v. 1, p. 399-416, 2013.

PAULO, V. I. M.; HERNÁNDEZ, E. P. Magnetization curves and atomic disorder in $\mathrm{La}_{2 / 3} \mathrm{Sr}_{1 / 3} \mathrm{MnO}_{3}$ manganite. Materials Research Express, v. 1, p. 01-02, 2018.

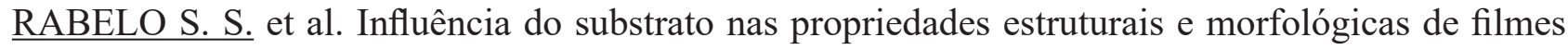
de manganita de lantânio dopados com estrôncio depositados por spray-pirólise. Matéria (Rio de Janeiro), v. 12, p. 44-53, 2007.

SHIMAKAWA, Y. et al. A variable-emittance radiator based on a metal-insulator transition of (La,Sr) $\mathrm{MnO}_{3}$ thin films. Applied Physics Letters, v. 80, n. 25, 2002.

SMESTAD, G. P.; STEINFELD, A. Photochemical and thermochemical production of solar fuels from $\mathrm{H}_{2} \mathrm{O}$ and $\mathrm{CO}_{2}$ using metal oxide catalysts. Ind. Eng. Chem. Res. v. 51, n. 31, p. 11828-11840, 2012.

STAMBOULI, A. B.; TRAVERSA, E. Solid oxide fuel cells (SOFCs): A review of an environmentally clean and efficient source of energy. Renewable and Sustainable Energy Reviews, v. 6, n. 5, p. 
TRAN. T. H. et al. Phase transition of $\mathrm{LaMnO}_{3}$ nanoparticles prepared by microwave assisted combustion method. Materials Science in Semiconductor Processing, v. 89, p. 121-125, 2019.

TAPOUK, F. A. et al. Synthesis of PAC- $\mathrm{LaFeO}_{3}-\mathrm{Cu}$ nanocomposites via sol-gel method for the photo catalytic degradation of humic acids under visible light irradiation. Journal of Environmental Chemical Engineering, v. 9, n. 4, 2021.

TURKY, A. O. et al. Optical, electrica and magnetic properties of lanthanum strontium manganite $\mathrm{La}_{1-\mathrm{x}} \mathrm{Sr}_{\mathrm{x}} \mathrm{MnO}_{3}$ synthesized through the citate combustion method. Physical Chemistry Chemical Physics, 2017.

TURKY, A. O. et al. An investigation of structure and electrical characteristics of lanthanum strontium manganite nanopowders with different $\mathrm{Sr}^{2+}$ ion concentrations. Particulate Science and Technology, v. 36, n. 7, p. 873-877, 2018.

WU, Q. H. et al. X-ray photoelectron spectroscopy of $\mathrm{La}_{0.5} \mathrm{Sr}_{0.5} \mathrm{MnO}_{3}$. Materials Letters, v. 59, n. 16, p. 1980-1983, 2005.

$\mathrm{XU}$, D. et al. Sensitive electrochemical detection of glucose based on electrospun $\mathrm{La}_{0.88} \mathrm{Sr}_{0.12} \mathrm{MnO}_{3}$ naono fibers modified electrode. Analytical Biochemistry, v. 489, p. 38-43, 2015.

YENSANO, R. et al. Fabrication and magnetic properties of electrospun $\mathrm{La}_{0.7} \mathrm{Sr}_{0.3} \mathrm{MnO}_{3}$ nanostructures. Journal of Superconductivity and Novel Magnetism, v. 27, n. 6, p. 1553-1560, 2014.

ZHI, M. et al. Electrospun $\mathrm{La}_{0.8} \mathrm{Sr}_{0.2} \mathrm{MnO}_{3}$ nanofibers for a high-temperature electrochemical carbon monoxide sensor. Nanotechnology, v. 23, n. 30, 2012.

ZHUANG, Y. et al. Microstructure and elastic properties of $\mathrm{BaTiO}_{3}$ nanofibers sintered in various atmospheres. Ceramics International, v. 44, n. 2, p. 2426-2431, 2018. 


\section{Índice Remissivo}

A

Ácido cítrico 11, 12, 13, 14, 15, 16, 19, 20, 22, 23, 25, 35, 37, 39, 41, 45, 51, 52, 53

Ácido cítrico/cátions metálicos 11, 12, 13, 14, 16, 25, 35, 37, 45, 52, 53

Água residual 11, 15, 23

Álcool etílico 11, 12, 27, 28, 29, 30, 31, 53

Álcool isopropílico 27, 28, 30, 31

Alumínio 12, 13, 14, 20, 27, 28, 30, 31

Ambiente fisiológicos 45

Ambientes químicos 12, 19

Análise térmica 11, 12, 19, 21, 27, 29

Aplicações tecnológicas 11, 20, 68

Assistência à saúde humana 35

\section{B}

Beta fosfato tricálcico ( $\beta$-tcp) 35,39

Bioatividade 35, 45, 47, 49

Biocerâmicas 35, 45, 46, 47

Biocompatibilidade 37, 45, 46, 47, 49

Biomateriais 45, 46, 47, 50

Biomaterial cerâmico 35

$\mathrm{C}$

Calcinação 12, 19, 20, 21, 24, 27, 29, 30, 31, 37, 51

Cátions metálicos 11, 13, 14, 16, 19, 20, 35, 39, 41, 52

Cerâmicas perovskitas 61

Ciência/indústria 61,63

Condutividade térmica 12, 19

Cristalização da mulita 11, 14, 16, 19, 21, 24, 27, 31

$\mathrm{D}$

Decomposição dos componentes 11, 15, 23

Densidade 12, 19, 48, 63

Difração de raios x (drx) 11, 27, 29, 35, 38

E

Engenharia de tecidos 35, 36

Escala de homogeneidade 27

Estabilidade 12, 19, 35, 46, 50, 53, 62

Estabilidade térmica 12, 19, 46, 53

Estrôncio 49, 61, 62, 63, 65, 66, 67, 68, 70

Expansão térmica 12,19

F 
Fase alfa-alumina 19

Fase alumina 11

Fase mulita 11, 19, 22, 29, 30

Fases cristalinas 19

Fases de fosfatos de cálcio 35

Fosfato de cálcio bifásico 35

G

Géis difásicos 27

Géis monofásicos 27

$\mathrm{H}$

Hidroxiapatita (hap) 35

Hipertermia magnética 61, 63

$\mathrm{L}$

Liberador de fármacos 61,63

M

Manganita de lantânio 61, 62, 63, 70

Material cerâmico 11

Material orgânico 11, 15, 23

Mecanismos de reação 19

Método pechini $11,12,13,14,15,16,17,19,20,24,25,37,39,41,45,47,51,52,53$

Método sol-gel 20, 27, 28, 31, 37

Microambiente fisiológico 35

Microscopia eletrônica de varredura (mev) 19, 21, 35, 38

Mulita 11, 19, 20, 27, 28

$\mathrm{N}$

Nanofibras 61

Nano/micrométricos e monólitos 61

Nanotecnologia 61, 63

Nível atômico 13, 27

$\mathrm{O}$

Organismo humano 45

Osseointegração 35,37

$\mathrm{P}$

Perda ou falha de tecidos ou órgãos 35

Processamento sol-gel 27

$\mathrm{R}$

Refratariedade e desempenho do material 19

Resistência mecânica $12,19,46$ 
Ressonância magnética 61, 63

$\mathrm{S}$

Silício 13, 14, 27, 31

Síntese 11, 12, 13, 14, 19, 20, 25, 27, 28, 37, 39, 45, 47, 50, 51, 52, 53, 63

Solvente 11, 19, 20, 21, 22, 24, 27, 28, 30, 31, 45, 53

$\mathrm{T}$

Tetrahidrofurano 19

Tipos de géis 27

Toxicidade $37,38,40,41,42,45,47$ 
EDITORA

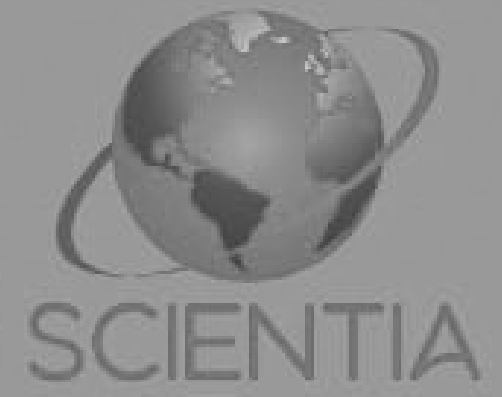

OMNIS SCIENTIA

editoraomnisscientia@gmail.com M https://editoraomnisscientia.com.br/ @editora_omnis_scientia (0) https://www.facebook.com/omnis.scientia.9 $\subsetneq$

+55 (87) 9656-3565 @ 
EDITORA

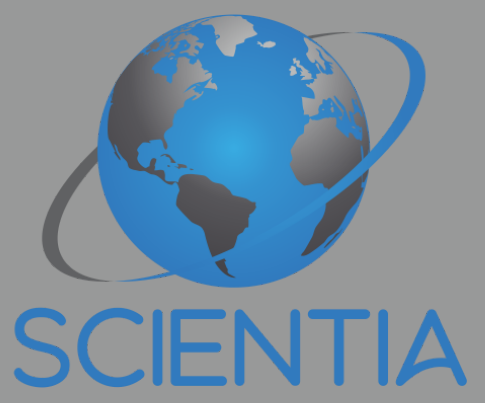

OMNIS SCIENTIA

editoraomnisscientia@gmail.com M https://editoraomnisscientia.com.br/ ( @editora_omnis_scientia (0) https://www.facebook.com/omnis.scientia.9 $\subsetneq$ +55 (87) 9656-3565 @ 